\title{
Analysis of Predictive Factors for Deterioration of Renal Function in Chronic Kidney Disease Patients
}

\author{
Keiichi Matsuzaki ${ }^{1,2 \#}$, Hitoshi Suzuki ${ }^{1 \#}$, Takashi Kobayashi ${ }^{1}$, Yoshio Shimizu ${ }^{1}$ and Yasuhiko Tomino ${ }^{1^{*}}$ \\ ${ }^{1}$ The Clinical Research Project for Intensive Therapy of Chronic Kidney Disease (CKD), Juntendo University Faculty of Medicine, Tokyo, Japan \\ ${ }^{2}$ Kyoto University Health Service, Kyoto, Japan \\ "These authors contributed equally to this work
}

\begin{abstract}
Chronic kidney disease (CKD) management requires a multidisciplinary approach. Although several treatment targets exist, the relationships between a number of clinical criteria and CKD progression have not been studied. Here, we investigated the association between renal dysfunction progression and a number of clinical parameters. We retrospectively enrolled 373 patients with mild impaired renal function indicated by a serum creatinine level > $2.0 \mathrm{mg} / \mathrm{dL}$ measured in 2012. We assessed clinical parameters both in 2009 and 2012, and analyzed whether each clinical parameter (e.g., hypertension, diabetes, dyslipidemia, and anemia) met therapeutic targets. We defined a $50 \%$ increase in serum creatinine level as baseline, and determined the progression and non-progression groups based on this definition. Systolic blood pressure (SBP), estimated glomerular filtration rate (eGFR), triglyceride, and urinary protein were significantly different between the progression and non-progression groups. The percentage of individuals in the non-progression group decreased with increasing proteinuria $(<0.2 \mathrm{~g} / \mathrm{gCr}: 83.3 \%,<0.3 \mathrm{~g} / \mathrm{gCr}: 82.1 \%,<0.5 \mathrm{~g} / \mathrm{gCr}$ : $78.3 \%,<1.0 \mathrm{~g} / \mathrm{gCr}: 72.8 \%$ ). In the multiple regression model, the number of clinical criteria achieved was significantly associated with renal progression. Moreover, the model including SBP, HbA1c, urinary protein, and triglyceride; e.g. intensive treatment, showed the strongest relationship (odds ratio $0.65,95 \%$ confidence interval $0.53-0.82, p<0.001$ ). To prevent renal dysfunction progression, treatment with renin-angiotensin system inhibitor and statin are not sufficient in CKD patients. Intensive treatment of SBP, $\mathrm{HbA1c}$, urinary protein, and triglyceride is essential. Even in patients with low eGFR, exacerbation of renal injuries was prevented with intensive treatment.
\end{abstract}

Keywords: Chronic kidney disease; Urinary protein; Blood pressure; Triglyceride; Predictive factor

\section{Introduction}

Recently, the increased number of patients with end-stage renal disease has become a major global health problem [1,2]. In 2002, the Kidney Disease Outcomes Quality Initiative proposed a new concept of renal disease known as chronic kidney disease (CKD) [2]. The association between CKD and adverse cardiovascular events has been established in several clinical studies [3,4]. Moreover, recent studies revealed that CKD is associated with dementia [5] and depression [6]. Intensive treatment for CKD is important to protect renal dysfunction and damage to other organs. Thus, the management of CKD improves patients' activities of daily living and quality of life. In 2007 and 2012, the Japanese Society of Nephrology (JSN) proposed a guide for the management of CKD for nephrologists and primary care physicians in Japan [7]. In this guide, the JSN proposed several therapeutic targets for each clinical criterion, such as hypertension, diabetes, dyslipidemia, and anemia. Furthermore, several studies revealed that urinary protein (UP) is one of the major factors affecting CKD progression [8]. The objective of the present study was to investigate the association between renal progression and the clinical criteria of CKD management. In addition, we showed that the CKD guide was useful in the prevention of CKD progression in our cohort.

\section{Methods}

\section{Study design and subjects}

This study is a retrospective cohort study conducted in the outpatient clinic of Department of Nephrology and Hypertension at Juntendo University Hospital. CKD patients with serum creatinine (S-Cr) level $\geq 2.0 \mathrm{mg} / \mathrm{dl}$ in $2012(\mathrm{n}=382)$ were eligible for present study (Figure 1). We assessed serum creatinine both in 2009 and in 2012. Of the 382 patients, 9 patients were excluded because patients underwent the dialysis from 2009 to 2012. Moreover, we defined the patients who has serum creatinine level $\leq 2.0 \mathrm{mg} / \mathrm{dl}$ in 2009 as the "mild impaired renal function group". This study was approved by the Ethical Committee of Juntendo University Hospital (13-021), and all participants gave written informed consent.

\section{Clinical data and definitions of outcomes}

We collected data on baseline clinical characteristics from a review of medical records in both 2009 and 2012. Clinical characteristics comprised age, gender, height, body weight, systolic blood pressure (SBP), diastolic blood pressure, albumin level, S-Cr level, estimated glomerular filtration rate (eGFR) estimated by the modified modification of diet in renal disease equation $(\mathrm{eGFR}=194 \times$ age [year] ${ }^{0.287} \times \mathrm{S}-\mathrm{Cr}[\mathrm{mg} / \mathrm{dL}]^{-1.094}(\times 0.739$ if female $\left.)\right)$ [9]. Levels of plasma glucose, low-density lipoprotein (LDL) cholesterol, high-density lipoprotein (HDL) cholesterol, triglyceride (TG), uric, hemoglobin ( $\mathrm{Hb})$, serum calcium, serum phosphorus, serum potassium, UP, and urinary occult blood on dipstick analysis were also comprised. We collected data on therapeutic interventions as follows: use of erythropoiesis stimulating agents, antihypertensive drugs including RAS-I, statins, anti-diabetic drugs, insulin, spherical adsorptive carbon, and sodium bicarbonate. We defined that a primary outcome that was the $50 \%$ increase in S-Cr as

*Corresponding author: Yasuhiko Tomino, University Faculty of Medicine, 2-1-1 Hongo, Bunkyo-ku, Tokyo, Japan, 113-8421, Tel/Fax: +81-3-5802-1065; E-mail: yasu@juntendo.ac.jp

Received: March 10, 2016; Accepted: March 19, 2016; Published: March 26 2016

Citation: Matsuzaki K, Suzuki H, Kobayashi T, Shimizu Y, Tomino Y (2016) Analysis of Predictive Factors for Deterioration of Renal Function in Chronic Kidney Disease Patients. J Nephrol Ther 6: 240. doi:10.4172/2161-0959.1000240

Copyright: @ 2016 Matsuzaki K, et al. This is an open-access article distributed under the terms of the Creative Commons Attribution License, which permits unrestricted use, distribution, and reproduction in any medium, provided the original author and source are credited. 
baseline, and determined the progression and non-progression groups based on this value. We defined patients who had mild impaired renal function ( $\mathrm{S}-\mathrm{Cr} \leq 2.0 \mathrm{mg} / \mathrm{dL}$ ) at 2009 as a separate subgroup.

\section{Management indicators for each parameter}

Based on the clinical practice guidebook for the diagnosis and treatment of CKD from 2012 [7], we determined the criteria for the management of CKD (i.e. SBP $\leq 130 \mathrm{mmHg}, \mathrm{HbAlc} \leq 6.9 \%$, LDL cholesterol $\leq 120 \mathrm{mg} / \mathrm{dL})$. We also determined the criteria of UP $(<$ $0.3 \mathrm{~g} / \mathrm{gCr})$ and TG level $(\leq 150 \mathrm{mg} / \mathrm{dL})$. We categorized the patients according to these criteria in both 2009 and 2012.

\section{Statistical Analysis}

We performed statistical analyses using Stata Version 13 (StataCorp, Collage Station, TX, USA). Normally distributed continuous variables were expressed as means with standard deviations and compared using Student's t-test. Non-normally distributed continuous variables were expressed as medians (interquartile ranges) and compared using the Mann-Whitney U test. Categorical variables were expressed as numbers (proportions) and analyzed using the chi-square test or Fisher's exact test. Logistic regression was used to calculate the odds ratio (OR) for the association between the number of clinical criteria satisfied and the percentage of patients with progression of renal dysfunction. All probability values were two-tailed, and all confidence intervals were compared at the $95 \%$ level.

\section{Results}

\section{Clinical characteristics and demographic data of the enrolled CKD patients}

We compared baseline characteristics between the progression and non-progression groups (Table 1). SBP was significantly lower (129.5 \pm $17.2 \mathrm{mmHg}$ vs. $140.4 \pm 73.4 \mathrm{mmHg}$; $\mathrm{p}<0.01)$ in the non-progression group. TG levels were also significantly lower in the non-progression group $(145.6 \pm 68.1 \mathrm{mg} / \mathrm{dL}$ vs. $171.5 \pm 118.3 \mathrm{mg} / \mathrm{dL} ; \mathrm{p}=0.01)$. Renal function in the non-progression group was significantly lower than that in the progression group (S-Cr: $2.36 \pm 0.86 \mathrm{mg} / \mathrm{dL}$ vs. $2.15 \pm 0.96$ $\mathrm{mg} / \mathrm{dL} ; \mathrm{p}<0.03$, eGFR: $23.8 \pm 7.8 \mathrm{~mL} / \mathrm{min} / 1.73 \mathrm{~m}^{2}$ vs. $29.4 \pm 15.0$ $\left.\mathrm{mL} / \mathrm{min} / 1.73 \mathrm{~m}^{2} ; \mathrm{p}<0.01\right)$. Median UP level was significantly lower in the non-progression group (0.69 vs. $1.45 ; \mathrm{p}<0.01)$. No significant differences were observed with respect to $\mathrm{HbA1c}$, LDL cholesterol, $\mathrm{HDL}$ cholesterol, $\mathrm{Hb}$, and ratio of medication with renin-angiotensin system inhibitor (RAS-I) and statin between the two groups.

\section{Analysis of parameters associated with renal deterioration in patients with mild impaired renal function}

The baseline characteristics of the patients with mild impaired renal function are shown in (Table 2) $(n=163)$. SBP, S-Cr, eGFR, and UP were significantly different between the non-progression and progression groups. In patients in the non-progression group, serum TG levels were lower than in patients in the progression group (150.2 \pm 68.6 vs. 179.4 $\pm 117.8 ; \mathrm{p}=0.07)$. Levels of HbA1c, LDL cholesterol, and $\mathrm{Hb}$, and ratio of medication with RAS-I and statin were not significantly different between the two groups.

\section{The amount of urinary protein was a major factor for renal progression}

We evaluated the association between UP level and CKD progression in both 2009 and 2012 (Figure 2). About 83\% of patients who achieved the criterion of $<0.2 \mathrm{~g} / \mathrm{gCr}$ in 2009 and $2012 \mathrm{did}$ not experience a decline in renal function. The percentage of patients with

CKD patients with $\mathrm{S}-\mathrm{Cr} \geq 2 \mathrm{mg} / \mathrm{dl}$ in $2012(\mathrm{n}=382)$

Excluded the patients underwent the renal replacement therapy in $2012(\mathrm{n}=9)$

373 patients were enrolled in this study (Table 1)

- Serum creatinine did not reach $50 \%$ increase in baseline $(n=173)$ (Non-progression group)

- Serum creatinine reached $50 \%$ increase in baseline $(\mathrm{n}=200)$ (Progression group)

Excluded the patients severely impaired renal function $(\mathrm{S}-\mathrm{Cr}>2 \mathrm{mg} / \mathrm{dl})$ in 2009

Sub analysis

163 patients had mild impaired renal function $(\mathrm{S}-\mathrm{Cr} \geq 2.0 \mathrm{mg} / \mathrm{dl}$ ) in 2009 (Table 2)

- Serum creatinine did not reach $50 \%$ increase in baseline $(n=66)$

(Non-progression group)

- Serum creatinine reached $50 \%$ increase in baseline $(n=97)$

- (Progression group)

Figure 1: Flow diagram with inclusion/exclusion criteria. 
Citation: Matsuzaki K, Suzuki H, Kobayashi T, Shimizu Y, Tomino Y (2016) Analysis of Predictive Factors for Deterioration of Renal Function in Chronic Kidney Disease Patients. J Nephrol Ther 6: 240. doi:10.4172/2161-0959.1000240

Page 3 of 7

\begin{tabular}{|c|c|c|c|c|}
\hline & All patients & Non-progression group & Progression group & $p$-value \\
\hline & $(n=373)$ & $(n=173)$ & $(n=200)$ & \\
\hline Age (years) & $66.1( \pm 13.9)$ & $66.8( \pm 14.1)$ & $65.5( \pm 13.8)$ & 0.39 \\
\hline Sex (male/female) & $278 / 95$ & $132 / 41$ & $146 / 54$ & 0.47 \\
\hline Height (cm) & $162.0( \pm 9.4)$ & $162.1( \pm 9.0)$ & $161.8( \pm 9.7)$ & 0.78 \\
\hline Body weight (kg) & $61.3( \pm 13.0)$ & $60.4( \pm 12.0)$ & $62.1( \pm 13.07)$ & 0.23 \\
\hline $\mathrm{SBP}(\mathrm{mmHg})$ & $135.2( \pm 54.8)$ & $129.5( \pm 17.2)$ & $140.4( \pm 73.4)$ & $<0.01$ \\
\hline $\mathrm{DBP}(\mathrm{mmHg})$ & $72.8( \pm 11.9)$ & $72.4( \pm 10.7)$ & $73.1( \pm 12.9)$ & 0.55 \\
\hline Alb (g/dL) & $4.0( \pm 0.44)$ & $4.1( \pm 0.40)$ & $3.9( \pm 0.47)$ & $<0.01$ \\
\hline S-cre (mg/dL) & $2.25( \pm 0.92)$ & $2.36( \pm 0.86)$ & $2.15( \pm 0.96)$ & 0.03 \\
\hline eGFR (mL/min) & $26.8( \pm 12.5)$ & $23.8( \pm 7.8)$ & $29.4( \pm 15.0)$ & $<0.01$ \\
\hline $\mathrm{BS}(\mathrm{mg} / \mathrm{dL})$ & $113.7( \pm 37.9)$ & $112.2( \pm 41.9)$ & $115.0( \pm 34.0)$ & 0.48 \\
\hline $\mathrm{HbA1c}(\%)$ & $6.0( \pm 0.9)$ & $6.0( \pm 0.8)$ & $6.0( \pm 0.9)$ & 0.43 \\
\hline LDL-cho (mg/dL) & $101.3( \pm 29.2)$ & $99.3( \pm 26.8)$ & $103.0( \pm 31.0)$ & 0.22 \\
\hline HDL-cho (mg/dL) & $50.6( \pm 16.0)$ & $49.7( \pm 15.3)$ & $51.3( \pm 16.5)$ & 0.31 \\
\hline TG (mg/dL) & $159.5( \pm 99.1)$ & $145.6( \pm 68.1)$ & $171.5( \pm 118.3)$ & 0.01 \\
\hline UA (mg/dL) & $6.9( \pm 1.3)$ & $6.9( \pm 1.2)$ & $7.0( \pm 1.3)$ & 0.22 \\
\hline $\mathrm{Hb}(\mathrm{g} / \mathrm{dL})$ & $11.8( \pm 1.8)$ & $11.9( \pm 1.7)$ & $11.7( \pm 1.8)$ & 0.24 \\
\hline $\mathrm{Ca}(\mathrm{mg} / \mathrm{dL})$ & $9.2( \pm 0.7)$ & $9.3( \pm 0.6)$ & $9.1( \pm 0.7)$ & 0.05 \\
\hline $\mathrm{Pi}(\mathrm{mg} / \mathrm{dL})$ & $3.5( \pm 0.8)$ & $3.5( \pm 0.9)$ & $3.5( \pm 0.7)$ & 0.67 \\
\hline $\mathrm{K}(\mathrm{mg} / \mathrm{dL})$ & $4.7( \pm 0.5)$ & $4.7( \pm 0.5)$ & $4.7( \pm 0.5)$ & 0.82 \\
\hline UP (g/day) & $1.05(0.42-2.1)$ & $0.69(0.19-1.49)$ & $1.45(0.57-2.91)$ & $<0.01$ \\
\hline Hematuria (\%) & $128(34.5)$ & $52(30.4)$ & $76(38.0)$ & 0.12 \\
\hline Use of RAS-I (\%) & $282(75.6)$ & $126(72.8)$ & $156(78.0)$ & 0.24 \\
\hline Use of statin (\%) & $121(32.4)$ & $61(35.3)$ & $60(30.0)$ & 0.27 \\
\hline
\end{tabular}

Table 1: Baseline characteristics and clinical parameters of the patients.

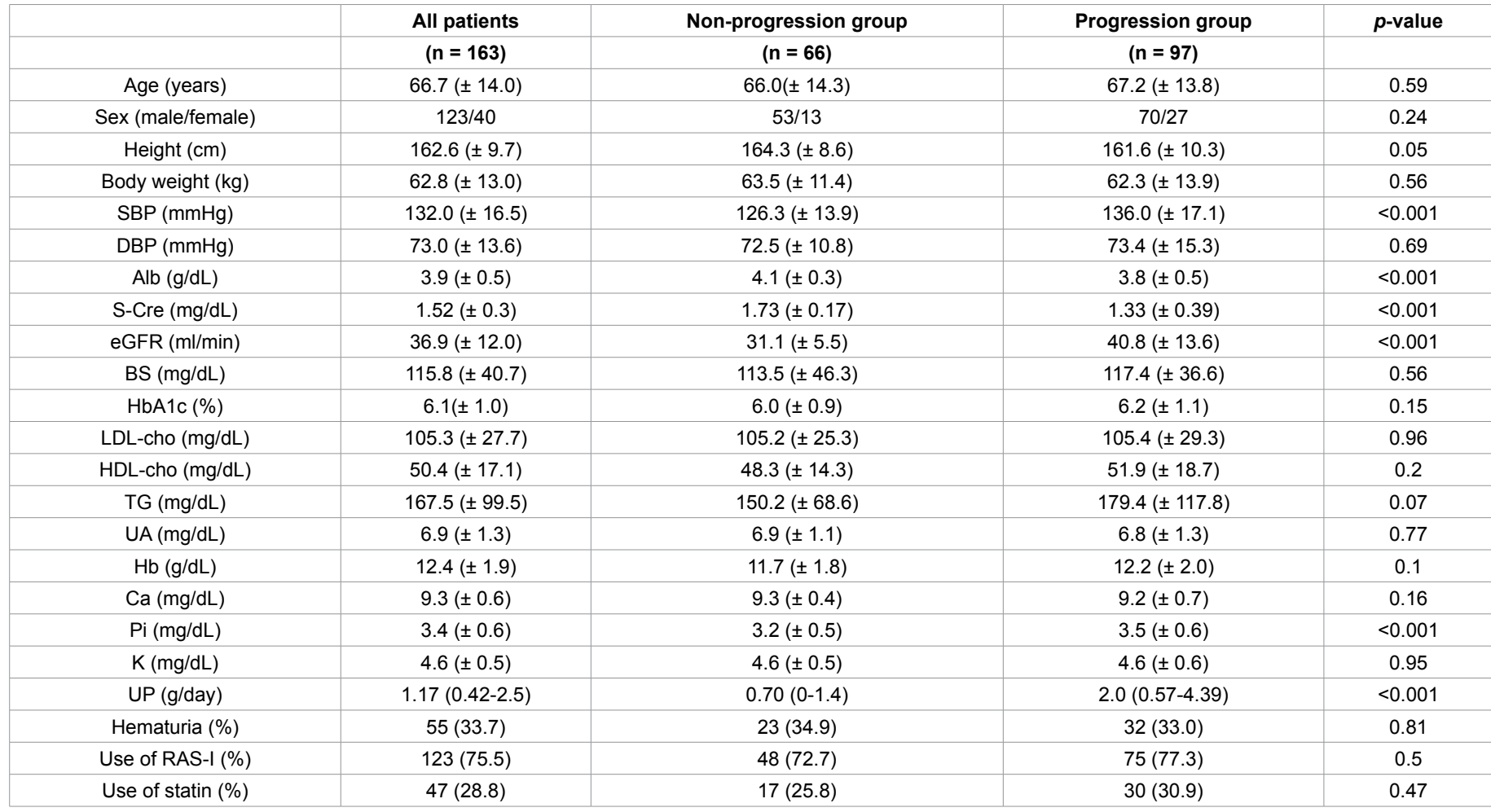

Table 2: Baseline characteristics of the patients with mild impaired renal function.

no progression of renal dysfunction decreased with increasing UP level (<0.2 g/gCr: 83.3\%, < 0.3 g/gCr: $82.1 \%,<0.5$ g/gCr: $78.3 \%,<1.0 \mathrm{~g} /$ gCr: $72.8 \%)$. Next, we evaluated whether the patients achieved the treatment criteria in 2009 and 2012. The percentage of patients who did not show a decline in renal function in each category of the CKD guide is shown in (Figure 3). Of the 105 patients who achieved the clinical criterion for SBP in both 2009 and 2012, 59 patients did not experience a decline in renal function (56.2\%). Of the 124 patients who achieved the criterion of $\mathrm{HbAlc}$ at both 2009 and 2012, 57 patients did not experience a decline in renal function (45.9\%). Of the 245 patients 


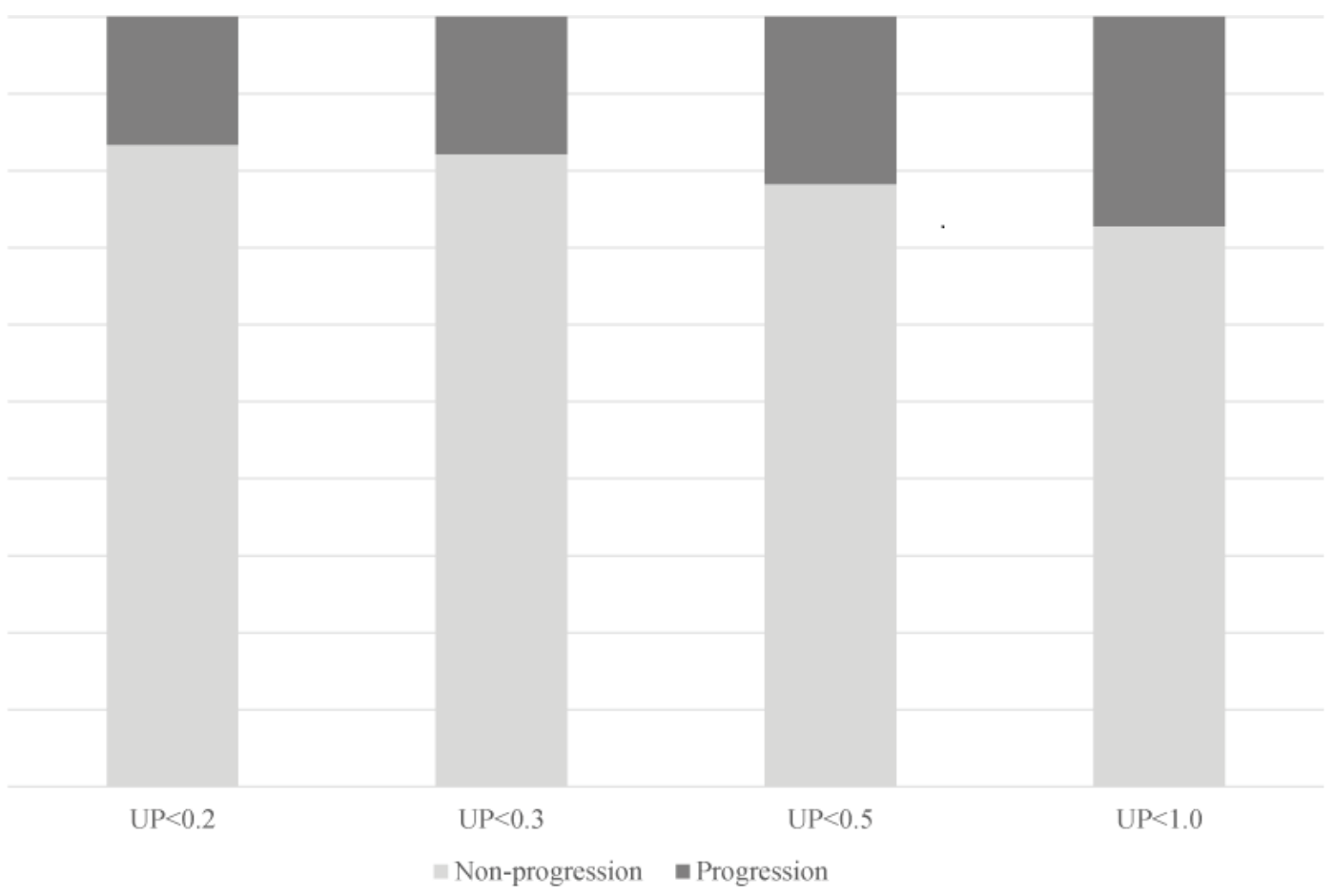

Figure 2: The amount of urinary protein associated with renal progression. The percentage of patients who did not show progression at each level of urinary protein The percentage of patients with no progression of renal dysfunction decreased with increasing levels of urinary protein.

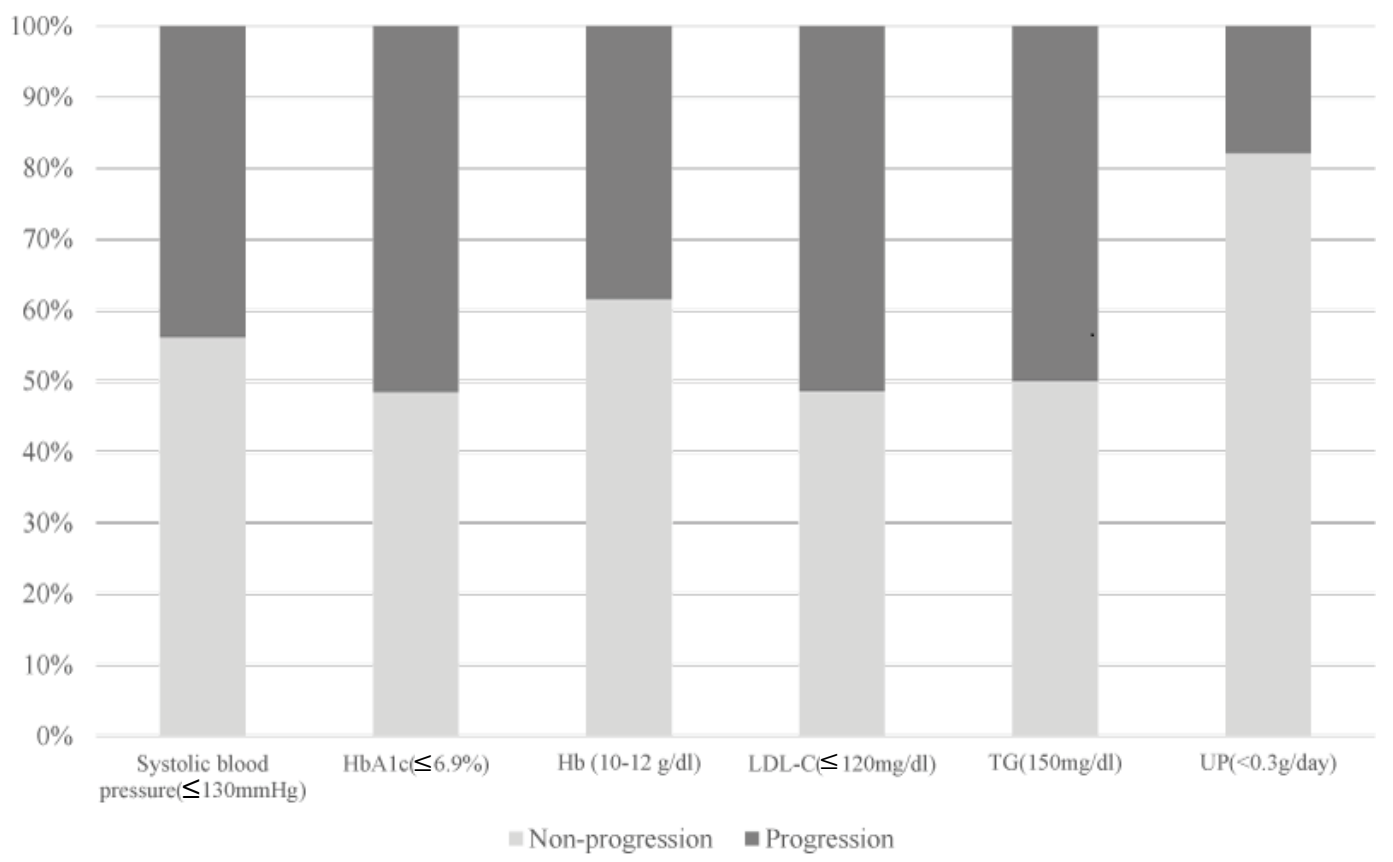

Figure 3: The contribution of each clinical criterion in the progression of renal function. The percentage of patients with no decline in renal function in each category of the CKD guide. The percentages of patients who achieved the clinical targets for systolic blood pressure and hemoglobin level were over $50 \%$.

who achieved the criterion for LDL cholesterol at both 2009 and 2012 119 patients did not experience a decline in renal function (48.5\%). Of the 166 patients who achieved the criterion for TG at both 2009 and 2012,83 patients did not experience a decline in renal function $(50.0 \%)$.
Of the 28 patients who achieved the criterion for UP at both 2009 and 2012, 23 patients did not experience a decline in renal function $(82.1 \%)$

The number of achieved clinical criteria was a significant 


\section{factor for the prevention of renal function loss}

The numbers of achieved clinical criteria in 2009 is shown in (Figure 4). The ratio of patients in the non-progression group who achieved above three clinical criteria (SBP, HbAlc, LDL, and $\mathrm{Hb}$ : model 1) was $54 \%$, while the ratio of those patients in the progression group was $45 \%$ (Figure $4 \mathrm{a}$ ). The ratio of patients in the non-progression group who achieved above four criteria (model $1+$ TG: model 2) was $42 \%$, while the ratio of those patients in the progression group was $30 \%$ (Figure 4b). In model 3 including SBP, HbA1c, TG and Hb, the ratio of patients in the non-progression and progression groups who achieved above three criteria were $51 \%$ and $37 \%$, respectively (Figure $4 \mathrm{c}$ ). The ratio of patients in the non-progression and progression groups who met above three criteria (SBP, HbA1c, TG, UP: model 4) were $35 \%$ and $22 \%$, respectively (Figure $4 \mathrm{~d}$ ). In logistic regression analysis, the specific criteria achieve by patients was a significant factor for renal function dysfunction in each model (model 1: OR $0.71,95 \%$ confidence interval [CI] 0.57-0.88, p = 0.002; model 2: OR 0.75, 95\% CI 0.62-0.89, p = 0.001 ; model 3: OR 0.73, 95\% CI 0.59-0.89, $\mathrm{p}=0.002$; model 4: OR $0.65,95 \%$ CI $0.53-0.82, \mathrm{p}<0.001)$.

\section{Discussion}

In this retrospective cohort study, we enrolled 373 patients with CKD and evaluated major factors associated with the progression of CKD during a 3-year period. This study showed that high levels of SBP,
TG and UP, and low eGFR were key factors for progression of CKD, even though the ratio of treatment with RAS-I and stain were not significantly different between the progression and non-progression groups. Moreover, we found that the achieved numbers of clinical criteria was related to protection of renal dysfunction. This is the first study to investigate this association. Control of $\mathrm{BP}$ is fundamental to the treatment of patients with $\mathrm{CKD}$, and is relevant at all stages of the disease [10]. The aim of $\mathrm{BP}$ control is to reduce the risk of renal dysfunction and mortality. Several studies have suggested that BP control using mainly RAS-I slows the progression of renal dysfunction at all stages of CKD [11-13] In this study, 56.2\% of the non-progression group achieved the therapeutic target for BP in both 2009 and 2012. In contrast, only $23.0 \%$ of CKD patients in the progression group achieved the therapeutic target for BP in both 2009 and 2012.

Several randomized controlled trials and meta-analyses [14-17] have suggested that the intensive control of plasma glucose levels inhibits the progression of diabetic nephropathy in the early stage. According to the Japanese CKD guide [7], the therapeutic target of $\mathrm{HbA1c}$ is below $6.9 \%$. In this study, only $22.7 \%$ of patients in the nonprogression group did not achieve the therapeutic target for $\mathrm{HbAlc}$ in both 2009 and 2012. In contrast, in the progression group, $77.3 \%$ of patients did not achieve the therapeutic target for HbAlc in both 2009 and 2012.

CKD is a significant risk factor for the incidence of cardiovascular a) $\mathrm{SBP}, \mathrm{HbA1c}, \mathrm{Hb}, \mathrm{LDL}$

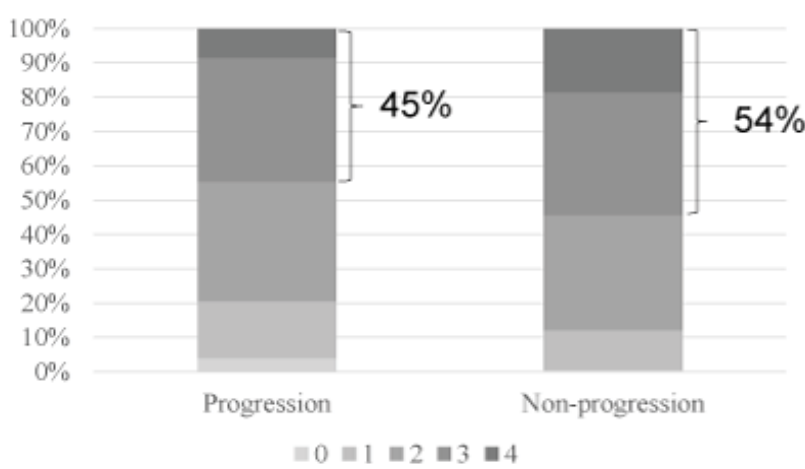

c) SBP, $\mathrm{HbA1c}, \mathrm{Hb}, \mathrm{TG}$

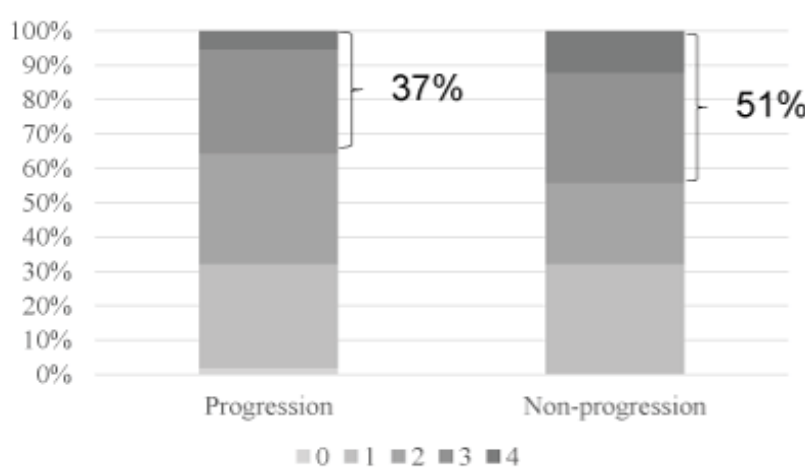

b) SBP, HbA1c, Hb, LDL, TG

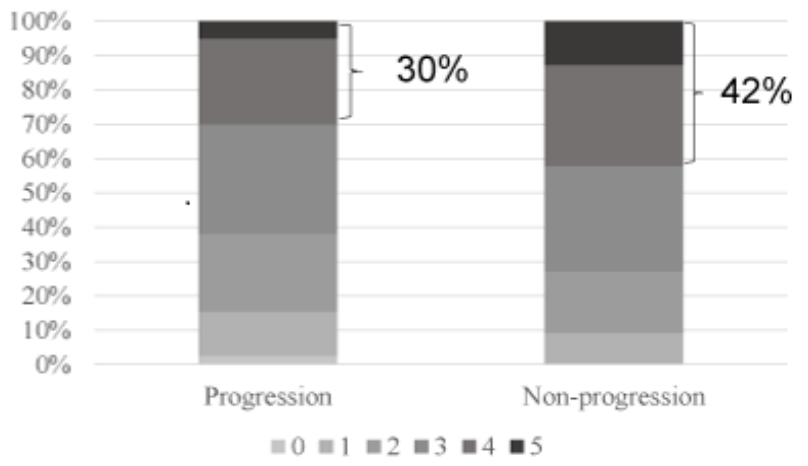

d) SBP, HbA1c, UP, TG

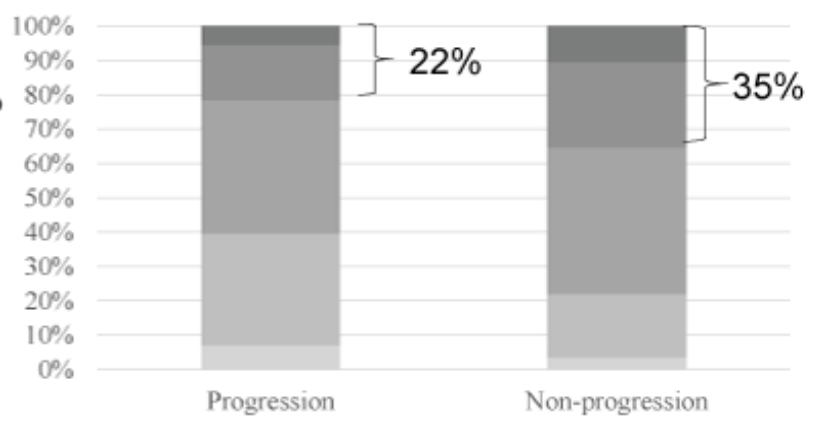

$=0=1=2=3=4$

Figure 4: The numbers of achieved clinical criteria in 2009. The number of achieved clinical criteria in the non-progression group was significantly greater than that in the progression group for all models. 
disease (CVD). Moreover, dyslipidemia is a significant risk factor for CKD progression and CVD. According to the Japanese CKD guide [7], the therapeutic target of LDL cholesterol is $<120 \mathrm{mg} / \mathrm{dL}$. In this study, the levels of LDL cholesterol were not significantly different between the progression and non-progression groups. In fact, $57.1 \%$ of patients in non-progression group did not achieve the therapeutic target for LDL cholesterol in both 2009 and 2012. These results do not confirm the importance of the therapeutic target for LDL cholesterol in CKD patients. Next, we investigated the clinical importance of serum TG levels. Although there was no significant difference in the level of LDL cholesterol between the progression and nonprogression groups, serum TG levels were significantly lower in the non-progression group. We determined the therapeutic target for TG level as $<150 \mathrm{mg} / \mathrm{dL}$ and performed the same evaluation (Figures $4 \mathrm{~b}$ and 4c). Several epidemiologic studies have shown that the incidence of CKD is associated with increased serum TG and LDL cholesterol levels, as well as decreased HDL cholesterol level. Since dyslipidemia induces arteriosclerosis in CKD patients, treating dyslipidemia is very important. However, the appropriate target lipid profile is unclear. Our results suggested that the management of TG levels is more important than those of LDL cholesterol. In the future, a large-scale clinical study is required to investigate the role of TG in the progression of CKD. Soluble tumorigenicity 2 (ST2) is suggested to be an early biomarker for CVD [18]. It is important to assess the serum levels of ST2 in progressive CKD patients in the future.

The Japanese CKD guide suggested the therapeutic targets for clinical criteria in CKD patients. However, to date, there is no evidence to suggest that the achieved numbers of clinical criteria is significant to protect progression of CKD. In order to investigate this hypothesis, we analyzed the achieved numbers of criteria in both groups in 2009 and 2012. The achieved numbers of clinical criteria was positively correlated with the maintenance of renal function (Figure $3, \mathrm{p}=0.04$ ). The logistic regression model clearly indicated that intensive treatment for BP, diabetes, high TG level, and proteinuria is essential to prevent progression of renal dysfunction (OR $0.6595 \%$ CI 0.53-0.82 p $<0.001$ : Table 3).

Importantly, eGFR in the non-progression group was significantly lower than that in the progression group at baseline (Table 1). However, mean eGFR at 2012 in the non-progression group was significantly higher than that in the progression group (19.7 \pm 0.44 vs. $12.6 \pm 0.47$, p $<0.001$ ). In the mild impaired group ( $\mathrm{S}-\mathrm{Cr} \leq 2.0 \mathrm{mg} / \mathrm{dL}$ in 2009$)$, eGFR at baseline did not predict the progression of CKD during the 3-year period of the study (Table 2). These results suggested that the intensive treatment of various clinical targets affected renal outcomes even in patients with low eGFR at baseline.

\section{Study Limitation}

This study has several limitations. First, we only included patients who could be followed up in our outpatient clinic in both 2009 and

\begin{tabular}{|c|c|c|c|}
\hline Model & OR & $\mathbf{9 5 \% C l}$ & $\boldsymbol{p}$-value \\
\hline Model 1 (SBP, HbA1c, Hb, LDL-C) & 0.71 & $0.57-0.88$ & 0.002 \\
\hline $\begin{array}{c}\text { Model 2 (SBP, HbA1c, Hb, LDL-C, } \\
\text { TG) }\end{array}$ & 0.75 & $0.62-0.89$ & 0.001 \\
\hline Model 3 (SBP, HbA1c, Hb, TG) & 0.73 & $0.59-0.89$ & 0.002 \\
\hline Model 4 (SBP, HbA1c, TG, UP) & 0.65 & $0.53-0.82$ & $<0.001$ \\
\hline
\end{tabular}

Table 3: Association between the achieved numbers of clinical criteria and renal progression.
2012. Therefore, some selection bias may have occurred. Second, the patients were administered various medicine in each clinical criteria. However, we could not estimate different efficacy in each treatment. Third, we defined the primary outcome as a $50 \%$ increase in S-Cr. However, we could not evaluate the cause of CKD in each patient.

\section{Conclusion}

In conclusion, we confirmed that hypertension, diabetes and amounts of UP are risk factors for the progression of CKD. However high serum TG levels also affected the deterioration of renal function. Importantly, reducing proteinuria had a strong effect on preventing CKD progression. Moreover, we detected that achieving more therapeutic criteria had a strong effect on protecting renal function, even in the case with treatment with RAS-I and statin. Together, these results suggest that intensive treatment is essential in patients with $\mathrm{CKD}$, regardless of baseline eGFR.

\section{Acknowledgements}

This study was supported by a grant in a part by The Clinical Research Project for Intensive Therapy of Chronic Kidney Disease: a collaborative project between Kowa Co. Ltd., Kyowa Hakko Kirin Co. Ltd., Mitsubishi Tanabe Pharma Corporation, and Novartis Pharma. The authors are grateful to Mr. Kaoru Sasao for his excellent clerical assistance.

\section{Declaration of Interest}

The authors report no conflicts of interest. The authors alone are responsible for the content and writing of the paper.

\section{References}

1. Lysaght MJ (2002) Maintenance dialysis population dynamics: current trends and long-term implications. J Am Soc Nephrol 13 Suppl 1: S37-40.

2. Tomino $Y$ (2014) Pathogenesis and treatment of chronic kidney disease: a review of our recent basic and clinical data. Kidney Blood Press Res 39: 450489.

3. National Kidney Foundation (2002) K/DOQI clinical practice guidelines fo chronic kidney disease: evaluation, classification, and stratification. Am J Kidney Dis 39: S1-266.

4. Sarnak MJ, Levey AS, Schoolwerth AC, Coresh J, Culleton B, et al. (2003) Kidney disease as a risk factor for development of cardiovascular disease: a statement from the American Heart Association Councils on Kidney in Cardiovascular Disease, High Blood Pressure Research, Clinical Cardiology and Epidemiology and Prevention. Hypertension 42: 1050-1065.

5. Chronic Kidney Disease Prognosis Consortium, Matsushita $\mathrm{K}$, van der Velde M, Astor BC, Woodward M, et al. (2010) Association of estimated glomerular filtration rate and albuminuria with all-cause and cardiovascular mortality in general population cohorts: a collaborative meta-analysis. Lancet 375: 2073 2081.

6. Miwa K, Tanaka M, Okazaki S, Furukado S, Yagita Y, et al. (2014) Chronic kidney disease is associated with dementia independent of cerebral smallvessel disease. Neurology 82: 1051-1057.

7. Lopes AA, Albert JM, Young EW, Satayathum S, Pisoni RL, et al. (2004) Screening for depression in hemodialysis patients: associations with diagnosis, treatment, and outcomes in the DOPPS. Kidney Int 66: 2047-2053.

8. Japan nephrology society (2012) [Special issue: Clinical practice guidebook for diagnosis and treatment of chronic kidney disease 2012]. Nihon Jinzo Gakkai Shi 54: 1034-1191.

9. Nohara N, lo H, Matsumoto M, Furukawa M, Okumura K, et al. (2015) Predictive factors associated with increased progression to dialysis in early chronic kidney disease (stage 1-3) patients. Clin Exp Nephrol .

10. Matsuo S, Imai E, Horio M, Yasuda Y, Tomita K, et al. (2009) Revised equations for estimated GFR from serum creatinine in Japan. Am J Kidney Dis 53: 982 992.

11. Jafar TH, Stark PC, Schmid CH, Landa M, Maschio G, et al. (2003) Progression of chronic kidney disease: the role of blood pressure control, proteinuria, and angiotensin-converting enzyme inhibition: a patient-level meta-analysis. Ann 
Citation: Matsuzaki K, Suzuki H, Kobayashi T, Shimizu Y, Tomino Y (2016) Analysis of Predictive Factors for Deterioration of Renal Function in Chronic Kidney Disease Patients. J Nephrol Ther 6: 240. doi:10.4172/2161-0959.1000240

Page 7 of 7

Intern Med 139: 244-252.

12. Duckworth W, Abraira C, Moritz T, Reda D, Emanuele N, et al. (2009) Glucose control and vascular complications in veterans with type 2 diabetes. $\mathrm{N}$ Engl $\mathrm{J}$ Med 360: 129-139

13. Peterson JC, Adler S, Burkart JM, Greene T, Hebert LA, et al. (1995) Blood pressure control, proteinuria, and the progression of renal disease. The Modification of Diet in Renal Disease Study. Ann Intern Med 123: 754-762.

14. Uzu T, Nakao K, Kume S, Araki H, Isshiki K, et al. (2012) High sodium intake is associated with masked hypertension in Japanese patients with type 2 diabetes and treated hypertension. Am J Hypertens 25: 1170-1174.

15. Ninomiya T, Kiyohara Y, Kubo M, Tanizaki Y, Doi Y, et al. (2005) Chronic kidney disease and cardiovascular disease in a general Japanese population: the Hisayama Study. Kidney Int 68: 228-236.

16. Hemmingsen B, Lund SS, Gluud C, Vaag A, Almdal T, et al. (2011) Intensive glycaemic control for patients with type 2 diabetes: systematic review with meta-analysis and trial sequential analysis of randomised clinical trials. BM 343: d6898.

17. Boussageon R, Bejan-Angoulvant T, Saadatian-Elahi M, Lafont S, Bergeonneau C, et al. (2011) Effect of intensive glucose lowering treatment on all-cause mortality, cardiovascular death, and microvascular events in type 2 diabetes: meta-analysis of randomised controlled trials. BMJ 343: d4169.

18. Ciccone MM, Cortese F, Gesualdo M, Riccardi R, Di Nunzio D, et al. (2013) A novel cardiac bio-marker: ST2: a review. Molecules 18: 15314-15328. 